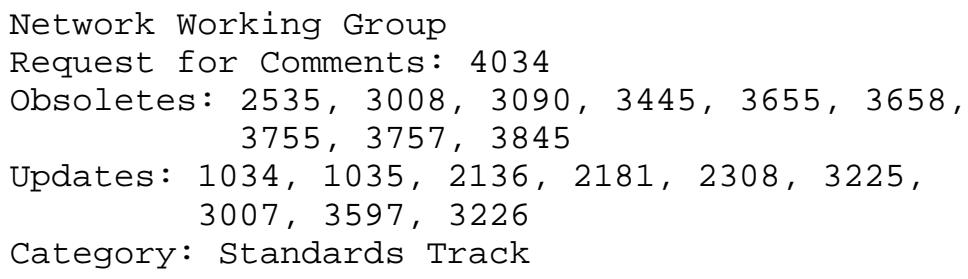

R. Arends Telematica Instituut

R. Austein

ISC

M. Larson Verisign

D. Massey Colorado state University

S. Rose

NIST

March 2005

\title{
Resource Records for the DNS Security Extensions
}

Status of This Memo

This document specifies an Internet standards track protocol for the Internet community, and requests discussion and suggestions for improvements. Please refer to the current edition of the "Internet Official Protocol Standards" (STD 1) for the standardization state and status of this protocol. Distribution of this memo is unlimited.

Copyright Notice

Copyright (C) The Internet Society (2005).

Abstract

This document is part of a family of documents that describe the DNS Security Extensions (DNSSEC). The DNS Security Extensions are a collection of resource records and protocol modifications that provide source authentication for the DNS. This document defines the public key (DNSKEY), delegation signer (DS), resource record digital signature (RRSIG), and authenticated denial of existence (NSEC) resource records. The purpose and format of each resource record is described in detail, and an example of each resource record is given.

This document obsoletes RFC 2535 and incorporates changes from all updates to RFC 2535. 
Table of Contents

1. Introduction . . . . . . . . . . . . . . . . . . . . . 3

1.1. Background and Related Documents . . . . . . . . . . 3

1.2. Reserved Words. . . . . . . . . . . . . . . 3

2. The DNSKEY Resource Record . . . . . . . . . . . . . . . . . 4

2.1. DNSKEY RDATA Wire Format . . . . . . . . . . . . . . . 4

2.1.1. The Flags Field. • • • • • • • • • • . . . 4

2.1.2. The Protocol Field. . . . . . . . . . . 5

2.1.3. The Algorithm Field. . . . . . . . . . . 5

2.1.4. The Public Key Field. . . . . . . . . . . 5

2.1.5. Notes on DNSKEY RDATA Design . . . . . . . 5

2.2. The DNSKEY RR Presentation Format. . . . . . . . . . . 5

2.3. DNSKEY RR Example • • • • . . . . . . . . . . . 6

3. The RRSIG Resource Record . . . . . . . . . . . . . . . 6

3.1. RRSIG RDATA Wire Format. . . . . . . . . . . . . . . 7

3.1.1. The Type Covered Field . . . . . . . . . . 7

3.1.2. The Algorithm Number Field . . . . . . . . 8

3.1.3. The Labels Field. . . . . . . . . . . 8

3.1.4. Original TTL Field . . . . . . . . . . . 8

3.1.5. Signature Expiration and Inception Fields. . . 9

3.1.6. The Key Tag Field. . . . . . . . . . . . . . . 9

3.1.7. The Signer's Name Field. . . . . . . . . . . 9

3.1.8. The Signature Field. . . . . . . . . . . . . 9

3.2. The RRSIG RR Presentation Format . . . . . . . . . . . 10

3.3. RRSIG RR Example . . . . . . . . . . . . . . . . 11

4. The NSEC Resource Record . . . . . . . . . . . . . . . . 12

4.1. NSEC RDATA Wire Format . . . . . . . . . . . . . . . 13

4.1.1. The Next Domain Name Field. . . . . . . . . 13

4.1.2. The Type Bit Maps Field. . . . . . . . . . 13

4.1.3. Inclusion of Wildcard Names in NSEC RDATA. . 14

4.2. The NSEC RR Presentation Format. . . . . . . . . . . . 14

4.3. NSEC RR Example. . . . . . . . . . . . . . . . . 15

5. The DS Resource Record . . . . . . . . . . . . . . . 15

5.1. DS RDATA Wire Format . . . . . . . . . . . . 16

5.1.1. The Key Tag Field. . . . . . . . . . . . 16

5.1.2. The Algorithm Field. . . . . . . . . . . 16

5.1.3. The Digest Type Field. . . . . . . . . . . 17

5.1.4. The Digest Field . . . . . . . . . . . . . . 17

5.2. Processing of DS RRs When Validating Responses. . . 17

5.3. The DS RR Presentation Format. . . . . . . . . . . 17

5.4. DS RR Example. • . . . . . . . . . . . . . . . 18

6. Canonical Form and Order of Resource Records. . . . . . . 18

6.1. Canonical DNS Name Order . . . . . . . . . . . . 18

6.2. Canonical RR Form. . . . . . . . . . . . . . . 19

6.3. Canonical RR Ordering within an RRset. . . . . . . . 20

7. IANA Considerations. . . . . . . . . . . . . . . . . . . . 20

8. Security Considerations. . . . . . . . . . . . . . . . 21 
9. Acknowledgements . . . . . . . . . . . . . . . . . 22

10. References . . . . . . . . . . . . . . . . . . . . . . 22

10.1. Normative References . . . . . . . . . . . . . . 22

10.2. Informative References . . . . . . . . . . . . . . 23

A. DNSSEC Algorithm and Digest Types. . . . . . . . . . . . . . 24

A.1. DNSSEC Algorithm Types . . . . . . . . . . . . . . 24

A.1.1. Private Algorithm Types. . . . . . . . . 25

A.2. DNSSEC Digest Types. • • • • • • • • • • • • . . 25

B. Key Tag Calculation. . . . . . . . . . . . . . . . . . 25

B.1. Key Tag for Algorithm 1 (RSA/MD5). . . . . . . . . . . 27

Authors' Addresses . . . . . . . . . . . . . . . . . . 28

Full Copyright Statement . . . . . . . . . . . . . . . 29

\section{Introduction}

The DNS Security Extensions (DNSSEC) introduce four new DNS resource record types: DNS Public Key (DNSKEY), Resource Record Signature (RRSIG), Next Secure (NSEC), and Delegation Signer (DS). This document defines the purpose of each resource record (RR), the $R R^{\prime} s$ RDATA format, and its presentation format (ASCII representation).

\subsection{Background and Related Documents}

This document is part of a family of documents defining DNSSEC, which should be read together as a set.

[RFC4033] contains an introduction to DNSSEC and definition of common terms; the reader is assumed to be familiar with this document. [RFC4033] also contains a list of other documents updated by and obsoleted by this document set.

[RFC4035] defines the DNSSEC protocol operations.

The reader is also assumed to be familiar with the basic DNS concepts described in [RFC1034], [RFC1035], and the subsequent documents that update them, particularly [RFC2181] and [RFC2308].

This document defines the DNSSEC resource records. All numeric DNS type codes given in this document are decimal integers.

\subsection{Reserved Words}

The key words "MUST", "MUST NOT", "REQUIRED", "SHALL", "SHALL NOT", "SHOULD", "SHOULD NOT", "RECOMMENDED", "MAY", and "OPTIONAL" in this document are to be interpreted as described in [RFC2119]. 


\section{The DNSKEY Resource Record}

DNSSEC uses public key cryptography to sign and authenticate DNS resource record sets (RRsets). The public keys are stored in DNSKEY resource records and are used in the DNSSEC authentication process described in [RFC4035]: A zone signs its authoritative RRsets by using a private key and stores the corresponding public key in a DNSKEY RR. A resolver can then use the public key to validate signatures covering the RRsets in the zone, and thus to authenticate them.

The DNSKEY RR is not intended as a record for storing arbitrary public keys and MUST NOT be used to store certificates or public keys that do not directly relate to the DNS infrastructure.

The Type value for the DNSKEY RR type is 48.

The DNSKEY RR is class independent.

The DNSKEY RR has no special TTL requirements.

\subsection{DNSKEY RDATA Wire Format}

The RDATA for a DNSKEY RR consists of a 2 octet Flags Field, a 1 octet Protocol Field, a 1 octet Algorithm Field, and the Public Key Field.

$\begin{array}{llllllllllllllllllllll}1 & 1 & 1 & 1 & 1 & 1 & 1 & 1 & 1 & 1 & 2 & 2 & 2 & 2 & 2 & 2 & 2 & 2 & 2 & 2 & 3 & 3\end{array}$

$\begin{array}{llllllllllllllllllllllllllllllll}0 & 1 & 2 & 3 & 4 & 5 & 6 & 7 & 8 & 9 & 0 & 1 & 2 & 3 & 4 & 5 & 6 & 7 & 8 & 9 & 0 & 1 & 2 & 3 & 4 & 5 & 6 & 7 & 8 & 9 & 0 & 1\end{array}$ +-+-+-+-+-+-+-+-+-+-+-+-+-+-+-+-+-+-+-+-+-+-+-+-+-+-+-t--t-+-+-+-+

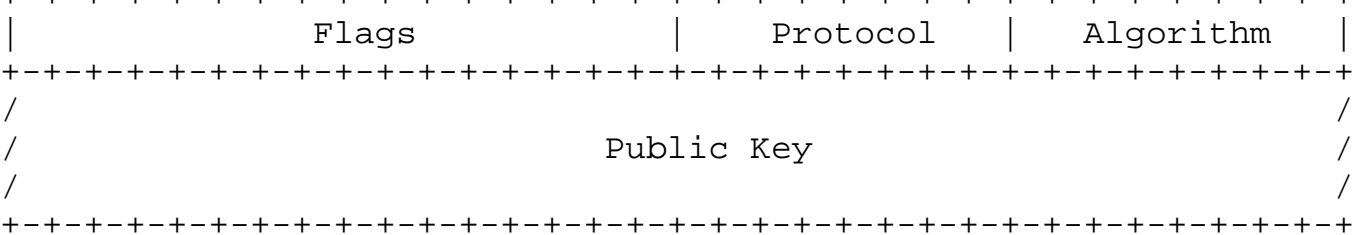

\subsubsection{The Flags Field}

Bit 7 of the Flags field is the Zone Key flag. If bit 7 has value 1, then the DNSKEY record holds a DNS zone key, and the DNSKEY RR's owner name MUST be the name of a zone. If bit 7 has value 0 , then the DNSKEY record holds some other type of DNS public key and MUST NOT be used to verify RRSIGs that cover RRsets.

Bit 15 of the Flags field is the Secure Entry Point flag, described in [RFC3757]. If bit 15 has value 1, then the DNSKEY record holds a key intended for use as a secure entry point. This flag is only 
intended to be a hint to zone signing or debugging software as to the intended use of this DNSKEY record; validators MUST NOT alter their behavior during the signature validation process in any way based on the setting of this bit. This also means that a DNSKEY RR with the SEP bit set would also need the Zone Key flag set in order to be able to generate signatures legally. A DNSKEY RR with the SEP set and the Zone Key flag not set MUST NOT be used to verify RRSIGs that cover RRsets.

Bits 0-6 and 8-14 are reserved: these bits MUST have value 0 upon creation of the DNSKEY RR and MUST be ignored upon receipt.

\subsubsection{The Protocol Field}

The Protocol Field MUST have value 3, and the DNSKEY RR MUST be treated as invalid during signature verification if it is found to be some value other than 3 .

2.1.3. The Algorithm Field

The Algorithm field identifies the public key's cryptographic algorithm and determines the format of the Public Key field. A list of DNSSEC algorithm types can be found in Appendix A.1

\subsubsection{The Public Key Field}

The Public Key Field holds the public key material. The format depends on the algorithm of the key being stored and is described in separate documents.

\subsubsection{Notes on DNSKEY RDATA Design}

Although the Protocol Field always has value 3, it is retained for backward compatibility with early versions of the KEY record.

\subsection{The DNSKEY RR Presentation Format}

The presentation format of the RDATA portion is as follows:

The Flag field MUST be represented as an unsigned decimal integer. Given the currently defined flags, the possible values are: 0, 256, and 257 .

The Protocol Field MUST be represented as an unsigned decimal integer with a value of 3 .

The Algorithm field MUST be represented either as an unsigned decimal integer or as an algorithm mnemonic as specified in Appendix A.1. 
The Public Key field MUST be represented as a Base64 encoding of the Public Key. Whitespace is allowed within the Base64 text. For a definition of Base64 encoding, see [RFC3548].

\subsection{DNSKEY RR Example}

The following DNSKEY RR stores a DNS zone key for example.com.

example.com. 86400 IN DNSKEY 25635 ( AQPSKmynfzW4kyBv015MUG2DeIQ3 $\mathrm{Cbl}+\mathrm{BBZH} 4 \mathrm{~b} / \mathrm{OPY} 1 \mathrm{kxkmvH} \mathrm{cZC} 8 \mathrm{no}$ kfzj31GajIQKY+5Cpt Lr 3buXA10h WqTkF7H6RfoRqXQeogmMHfpftf $6 z$ Mv1LyBUgia7za6zEzOJBOztyvhjL 742 iU/TpP SEDhm2SNKLi jfUppn1U $\operatorname{aNvV} 4 \mathrm{w}==$ )

The first four text fields specify the owner name, TTL, Class, and RR type (DNSKEY). Value 256 indicates that the Zone Key bit (bit 7) in the Flags field has value 1 . Value 3 is the fixed Protocol value. Value 5 indicates the public key algorithm. Appendix A.1 identifies algorithm type 5 as RSA/SHA1 and indicates that the format of the RSA/SHA1 public key field is defined in [RFC3110]. The remaining text is a Base64 encoding of the public key.

3. The RRSIG Resource Record

DNSSEC uses public key cryptography to sign and authenticate DNS resource record sets (RRsets). Digital signatures are stored in RRSIG resource records and are used in the DNSSEC authentication process described in [RFC4035]. A validator can use these RRSIG RRs to authenticate RRsets from the zone. The RRSIG RR MUST only be used to carry verification material (digital signatures) used to secure DNS operations.

An RRSIG record contains the signature for an RRset with a particular name, class, and type. The RRSIG RR specifies a validity interval for the signature and uses the Algorithm, the Signer's Name, and the Key Tag to identify the DNSKEY RR containing the public key that a validator can use to verify the signature.

Because every authoritative RRset in a zone must be protected by a digital signature, RRSIG RRs must be present for names containing a CNAME RR. This is a change to the traditional DNS specification [RFC1034], which stated that if a CNAME is present for a name, it is the only type allowed at that name. A RRSIG and NSEC (see Section 4) MUST exist for the same name as a CNAME resource record in a signed zone. 
The Type value for the RRSIG RR type is 46.

The RRSIG RR is class independent.

An RRSIG RR MUST have the same class as the RRset it covers.

The TTL value of an RRSIG RR MUST match the TTL value of the RRset it covers. This is an exception to the [RFC2181] rules for TTL values of individual RRs within a RRset: individual RRSIG RRs with the same owner name will have different TTL values if the RRsets they cover have different TTL values.

\subsection{RRSIG RDATA Wire Format}

The RDATA for an RRSIG RR consists of a 2 octet Type Covered field, a 1 octet Algorithm field, a 1 octet Labels field, a 4 octet Original TTL field, a 4 octet Signature Expiration field, a 4 octet Signature Inception field, a 2 octet Key tag, the Signer's Name field, and the Signature field.

$\begin{array}{llllllllllllllllllllll}1 & 1 & 1 & 1 & 1 & 1 & 1 & 1 & 1 & 1 & 2 & 2 & 2 & 2 & 2 & 2 & 2 & 2 & 2 & 2 & 3 & 3\end{array}$

$\begin{array}{llllllllllllllllllllllllllllllll}0 & 1 & 2 & 3 & 4 & 5 & 6 & 7 & 8 & 9 & 0 & 1 & 2 & 3 & 4 & 5 & 6 & 7 & 8 & 9 & 0 & 1 & 2 & 3 & 4 & 5 & 6 & 7 & 8 & 9 & 0 & 1\end{array}$

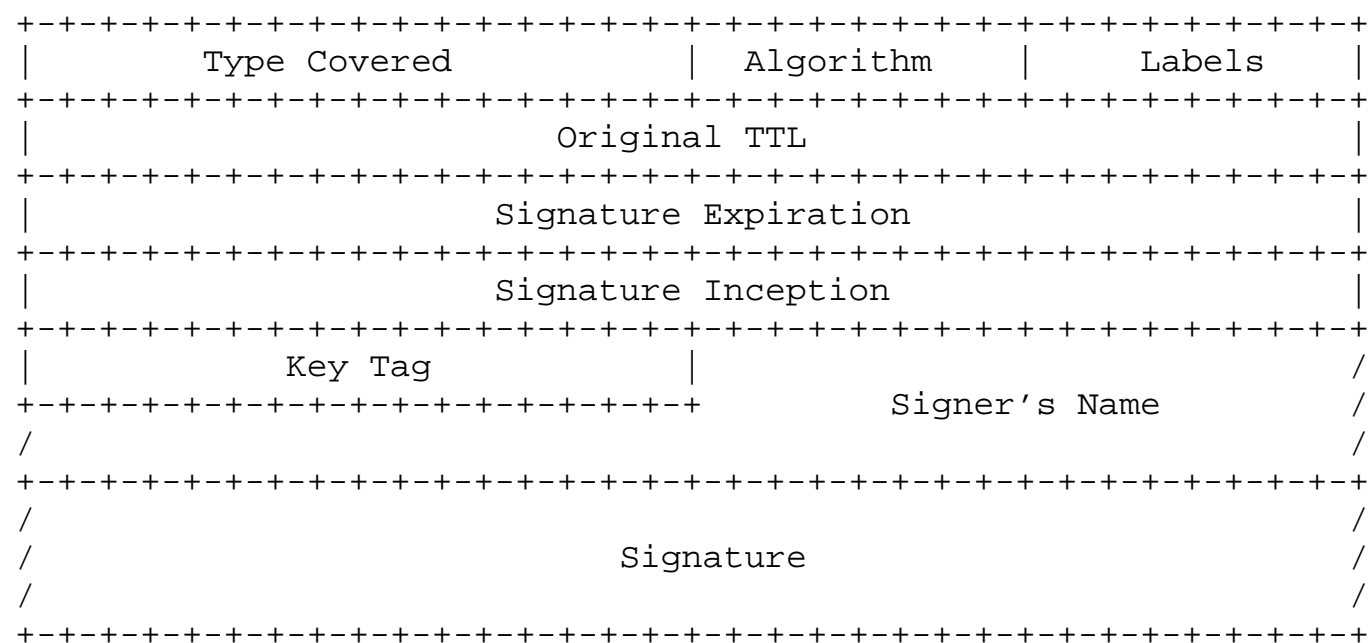

3.1.1. The Type Covered Field

The Type Covered field identifies the type of the RRset that is covered by this RRSIG record. 


\subsubsection{The Algorithm Number Field}

The Algorithm Number field identifies the cryptographic algorithm used to create the signature. A list of DNSSEC algorithm types can be found in Appendix A.1

\subsubsection{The Labels Field}

The Labels field specifies the number of labels in the original RRSIG RR owner name. The significance of this field is that a validator uses it to determine whether the answer was synthesized from a wildcard. If so, it can be used to determine what owner name was used in generating the signature.

To validate a signature, the validator needs the original owner name that was used to create the signature. If the original owner name contains a wildcard label ("*"), the owner name may have been expanded by the server during the response process, in which case the validator will have to reconstruct the original owner name in order to validate the signature. [RFC4035] describes how to use the Labels field to reconstruct the original owner name.

The value of the Labels field MUST NOT count either the null (root) label that terminates the owner name or the wildcard label (if present). The value of the Labels field MUST be less than or equal to the number of labels in the RRSIG owner name. For example, "www.example.com." has a Labels field value of 3, and "*.example.com." has a Labels field value of 2. Root (".") has a Labels field value of 0 .

Although the wildcard label is not included in the count stored in the Labels field of the RRSIG RR, the wildcard label is part of the RRset's owner name when the signature is generated or verified.

\subsubsection{Original TTL Field}

The Original TTL field specifies the TTL of the covered RRset as it appears in the authoritative zone.

The Original TTL field is necessary because a caching resolver decrements the TTL value of a cached RRset. In order to validate a signature, a validator requires the original TTL. [RFC4035] describes how to use the Original TTL field value to reconstruct the original TTL. 


\subsubsection{Signature Expiration and Inception Fields}

The Signature Expiration and Inception fields specify a validity period for the signature. The RRSIG record MUST NOT be used for authentication prior to the inception date and MUST NOT be used for authentication after the expiration date.

The Signature Expiration and Inception field values specify a date and time in the form of a 32-bit unsigned number of seconds elapsed since 1 January 1970 00:00:00 UTC, ignoring leap seconds, in network byte order. The longest interval that can be expressed by this format without wrapping is approximately 136 years. An RRSIG RR can have an Expiration field value that is numerically smaller than the Inception field value if the expiration field value is near the 32-bit wrap-around point or if the signature is long lived. Because of this, all comparisons involving these fields MUST use "Serial number arithmetic", as defined in [RFC1982]. As a direct consequence, the values contained in these fields cannot refer to dates more than 68 years in either the past or the future.

\subsubsection{The Key Tag Field}

The Key Tag field contains the key tag value of the DNSKEY RR that validates this signature, in network byte order. Appendix B explains how to calculate Key Tag values.

3.1.7. The Signer's Name Field

The Signer's Name field value identifies the owner name of the DNSKEY $R R$ that a validator is supposed to use to validate this signature. The Signer's Name field MUST contain the name of the zone of the covered RRset. A sender MUST NOT use DNS name compression on the Signer's Name field when transmitting a RRSIG RR.

\subsubsection{The Signature Field}

The Signature field contains the cryptographic signature that covers the RRSIG RDATA (excluding the Signature field) and the RRset specified by the RRSIG owner name, RRSIG class, and RRSIG Type Covered field. The format of this field depends on the algorithm in use, and these formats are described in separate companion documents.

\subsubsection{Signature Calculation}

A signature covers the RRSIG RDATA (excluding the Signature Field) and covers the data RRset specified by the RRSIG owner name, RRSIG class, and RRSIG Type Covered fields. The RRset is in canonical form (see Section 6), and the set $R R(1), \ldots R R(n)$ is signed as follows: 


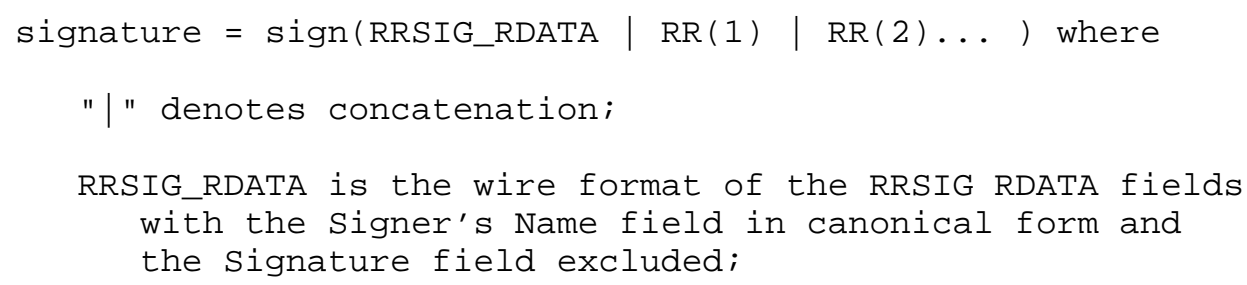

\subsection{The RRSIG RR Presentation Format}

The presentation format of the RDATA portion is as follows:

The Type Covered field is represented as an RR type mnemonic. When the mnemonic is not known, the TYPE representation as described in [RFC3597], Section 5, MUST be used.

The Algorithm field value MUST be represented either as an unsigned decimal integer or as an algorithm mnemonic, as specified in Appendix A. 1 .

The Labels field value MUST be represented as an unsigned decimal integer. 
The Original TTL field value MUST be represented as an unsigned decimal integer.

The Signature Expiration Time and Inception Time field values MUST be represented either as an unsigned decimal integer indicating seconds since 1 January 1970 00:00:00 UTC, or in the form YYYYMMDDHHmmSS in UTC, where:

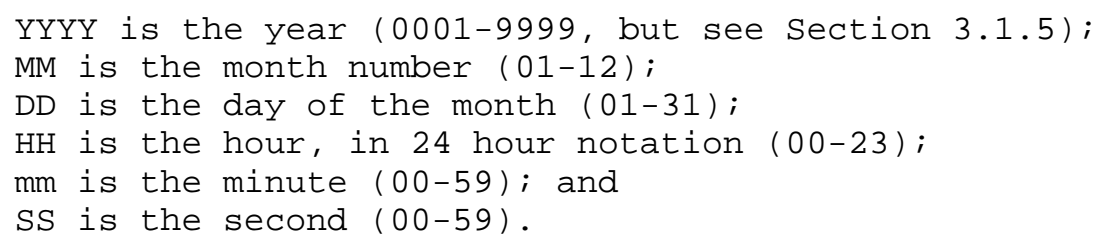

\subsection{RRSIG RR Example}

The following RRSIG RR stores the signature for the A RRset of host. example.com:

host.example.com. 86400 IN RRSIG A 538640020030322173103 ( 200302201731032642 example.com. oJB1W6WNGv+ldvQ3WDG0MQkg5IEhjRip8WTr PYGV07h108dUKGMeDPKi jVCHX3DDKdfb+v6o B9wfuh3DTJXUAfI/M0zmo/zz8bW0Rznl803t GNazPwQKkRN20XPXV6nwwfoXmJQbsLNrLfkG J5D 6 fwFm8nN+6pBzeDQfsS3Ap3o=) )

The first four fields specify the owner name, TTL, Class, and RR type (RRSIG). The "A" represents the Type Covered field. The value 5 identifies the algorithm used (RSA/SHA1) to create the signature. The value 3 is the number of Labels in the original owner name. The value 86400 in the RRSIG RDATA is the Original TTL for the covered A RRset. 20030322173103 and 20030220173103 are the expiration and 
inception dates, respectively. 2642 is the Key Tag, and example.com. is the Signer's Name. The remaining text is a Base64 encoding of the signature.

Note that combination of RRSIG RR owner name, class, and Type Covered indicates that this RRSIG covers the "host.example.com" A RRset. The Label value of 3 indicates that no wildcard expansion was used. The Algorithm, Signer's Name, and Key Tag indicate that this signature can be authenticated using an example.com zone DNSKEY RR whose algorithm is 5 and whose key tag is 2642 .

4. The NSEC Resource Record

The NSEC resource record lists two separate things: the next owner name (in the canonical ordering of the zone) that contains authoritative data or a delegation point NS RRset, and the set of RR types present at the NSEC RR's owner name [RFC3845]. The complete set of NSEC RRs in a zone indicates which authoritative RRsets exist in a zone and also form a chain of authoritative owner names in the zone. This information is used to provide authenticated denial of existence for DNS data, as described in [RFC4035].

Because every authoritative name in a zone must be part of the NSEC chain, NSEC RRs must be present for names containing a CNAME RR. This is a change to the traditional DNS specification [RFC1034], which stated that if a CNAME is present for a name, it is the only type allowed at that name. An RRSIG (see section 3) and NSEC MUST exist for the same name as does a CNAME resource record in a signed zone.

See [RFC4035] for discussion of how a zone signer determines precisely which NSEC RRs it has to include in a zone.

The type value for the NSEC RR is 47 .

The NSEC RR is class independent.

The NSEC RR SHOULD have the same TTL value as the SOA minimum TTL field. This is in the spirit of negative caching ([RFC2308]). 


\subsection{NSEC RDATA Wire Format}

The RDATA of the NSEC RR is as shown below:

$\begin{array}{llllllllllllllllllllll}1 & 1 & 1 & 1 & 1 & 1 & 1 & 1 & 1 & 1 & 2 & 2 & 2 & 2 & 2 & 2 & 2 & 2 & 2 & 2 & 3 & 3\end{array}$

$\begin{array}{llllllllllllllllllllllllllllllll}0 & 1 & 2 & 3 & 4 & 5 & 6 & 7 & 8 & 9 & 0 & 1 & 2 & 3 & 4 & 5 & 6 & 7 & 8 & 9 & 0 & 1 & 2 & 3 & 4 & 5 & 6 & 7 & 8 & 9 & 0 & 1\end{array}$

+-+-+-+-+-+-+-+-+-+-+-+-+-+-+-+-+-+-+-+-+-+-+-+-+-+-+-+-+-+-+-+-+

/ Next Domain Name /

+-+-+-+-+-+-+-+-+-+-+-+-+-+-+-+-+-+-+-+-+-+-+-+-+-+-+-+-+-+-+-+-+

/ Type Bit Maps /

+-+-+-+-+-+-+-+-+-+-+-+-+-+-+-+-+-+-+-+-+-+-+-+-+-+-+-+-+-+-+-+-+

4.1.1. The Next Domain Name Field

The Next Domain field contains the next owner name (in the canonical ordering of the zone) that has authoritative data or contains a delegation point NS RRset; see Section 6.1 for an explanation of canonical ordering. The value of the Next Domain Name field in the last NSEC record in the zone is the name of the zone apex (the owner name of the zone's SOA RR). This indicates that the owner name of the NSEC RR is the last name in the canonical ordering of the zone.

A sender MUST NOT use DNS name compression on the Next Domain Name field when transmitting an NSEC RR.

Owner names of RRsets for which the given zone is not authoritative (such as glue records) MUST NOT be listed in the Next Domain Name unless at least one authoritative RRset exists at the same owner name.

\subsubsection{The Type Bit Maps Field}

The Type Bit Maps field identifies the RRset types that exist at the NSEC RR's owner name.

The RR type space is split into 256 window blocks, each representing the low-order 8 bits of the 16-bit RR type space. Each block that has at least one active RR type is encoded using a single octet window number (from 0 to 255), a single octet bitmap length (from 1 to 32) indicating the number of octets used for the window block's bitmap, and up to 32 octets (256 bits) of bitmap.

Blocks are present in the NSEC RR RDATA in increasing numerical order.

Type Bit Maps Field = ( Window Block \# | Bitmap Length | Bitmap )+ where "|" denotes concatenation. 
Each bitmap encodes the low-order 8 bits of RR types within the window block, in network bit order. The first bit is bit 0 . For window block 0 , bit 1 corresponds to RR type 1 (A), bit 2 corresponds to RR type 2 (NS), and so forth. For window block 1, bit 1 corresponds to RR type 257, and bit 2 to RR type 258. If a bit is set, it indicates that an RRset of that type is present for the NSEC $\mathrm{RR}^{\prime} \mathrm{s}$ owner name. If a bit is clear, it indicates that no RRset of that type is present for the NSEC RR's owner name.

Bits representing pseudo-types MUST be clear, as they do not appear in zone data. If encountered, they MUST be ignored upon being read.

Blocks with no types present MUST NOT be included. Trailing zero octets in the bitmap MUST be omitted. The length of each block's bitmap is determined by the type code with the largest numerical value, within that block, among the set of RR types present at the NSEC RR's owner name. Trailing zero octets not specified MUST be interpreted as zero octets.

The bitmap for the NSEC RR at a delegation point requires special attention. Bits corresponding to the delegation NS RRset and the RR types for which the parent zone has authoritative data MUST be set; bits corresponding to any non-NS RRset for which the parent is not authoritative MUST be clear.

A zone MUST NOT include an NSEC RR for any domain name that only holds glue records.

4.1.3. Inclusion of Wildcard Names in NSEC RDATA

If a wildcard owner name appears in a zone, the wildcard label ("*") is treated as a literal symbol and is treated the same as any other owner name for the purposes of generating NSEC RRs. Wildcard owner names appear in the Next Domain Name field without any wildcard expansion. [RFC4035] describes the impact of wildcards on authenticated denial of existence.

\subsection{The NSEC RR Presentation Format}

The presentation format of the RDATA portion is as follows:

The Next Domain Name field is represented as a domain name.

The Type Bit Maps field is represented as a sequence of RR type mnemonics. When the mnemonic is not known, the TYPE representation described in [RFC3597], Section 5, MUST be used. 
4.3. NSEC RR Example

The following NSEC RR identifies the RRsets associated with alfa.example.com. and identifies the next authoritative name after alfa.example.com.

alfa.example.com. 86400 IN NSEC host.example.com. (

A MX RRSIG NSEC TYPE1234)

The first four text fields specify the name, TTL, Class, and RR type (NSEC). The entry host.example.com. is the next authoritative name after alfa.example.com. in canonical order. The A, MX, RRSIG, NSEC, and TYPE1234 mnemonics indicate that there are A, MX, RRSIG, NSEC, and TYPE1234 RRsets associated with the name alfa.example.com.

The RDATA section of the NSEC RR above would be encoded as:

\begin{tabular}{|c|c|c|c|c|c|c|c|}
\hline $0 \times 04$ & ${ }^{\prime} \mathrm{h}^{\prime}$ & ${ }^{\prime}$ & ${ }^{\prime} \mathrm{S}^{\prime}$ & $' t^{\prime}$ & & & \\
\hline $0 \times 07$ & $e^{\prime}$ & ${ }^{\prime} x^{\prime}$ & $' a^{\prime}$ & ${ }^{\prime} \mathrm{m}^{\prime}$ & ${ }^{\prime} \mathrm{p}^{\prime}$ & ${ }^{\prime} 1^{\prime}$ & $' e^{\prime}$ \\
\hline $0 \times 03$ & ${ }^{\prime} C^{\prime}$ & ${ }^{\prime} \mathrm{O}^{\prime}$ & $' \mathrm{~m}^{\prime}$ & $0 \times 00$ & & & \\
\hline $0 \times 00$ & $0 \times 06$ & $0 \times 40$ & $0 \times 01$ & $0 \times 00$ & $0 \times 00$ & $0 \times 00$ & $0 \times 03$ \\
\hline $0 \times 04$ & $0 \times 1 b$ & $0 \times 00$ & $0 \times 00$ & $0 \times 00$ & $0 \times 00$ & $0 \times 00$ & $0 \times 00$ \\
\hline $0 \times 00$ & $0 \times 00$ & $0 \times 00$ & $0 \times 00$ & $0 \times 00$ & $0 \times 00$ & $0 \times 00$ & $0 \times 00$ \\
\hline $0 \times 00$ & $0 \times 00$ & $0 \times 00$ & $0 \times 00$ & $0 \times 00$ & $0 \times 00$ & $0 \times 00$ & $0 \times 00$ \\
\hline $0 \times 00$ & $0 \times 00$ & $0 \times 00$ & $0 \times 00$ & $0 \times 20$ & & & \\
\hline
\end{tabular}

Assuming that the validator can authenticate this NSEC record, it could be used to prove that beta.example.com does not exist, or to prove that there is no AAAA record associated with alfa.example.com. Authenticated denial of existence is discussed in [RFC4035].

5. The DS Resource Record

The DS Resource Record refers to a DNSKEY RR and is used in the DNS DNSKEY authentication process. A DS RR refers to a DNSKEY RR by storing the key tag, algorithm number, and a digest of the DNSKEY RR. Note that while the digest should be sufficient to identify the public key, storing the key tag and key algorithm helps make the identification process more efficient. By authenticating the DS record, a resolver can authenticate the DNSKEY RR to which the DS record points. The key authentication process is described in [RFC4035].

The DS RR and its corresponding DNSKEY RR have the same owner name, but they are stored in different locations. The DS RR appears only on the upper (parental) side of a delegation, and is authoritative data in the parent zone. For example, the DS RR for "example.com" is stored in the "com" zone (the parent zone) rather than in the 
"example.com" zone (the child zone). The corresponding DNSKEY RR is stored in the "example.com" zone (the child zone). This simplifies DNS zone management and zone signing but introduces special response processing requirements for the DS RR; these are described in [RFC4035].

The type number for the DS record is 43.

The DS resource record is class independent.

The DS RR has no special TTL requirements.

5.1. DS RDATA Wire Format

The RDATA for a DS RR consists of a 2 octet key Tag field, a 1 octet Algorithm field, a 1 octet Digest Type field, and a Digest field.

$\begin{array}{llllllllllllllllllllll}1 & 1 & 1 & 1 & 1 & 1 & 1 & 1 & 1 & 1 & 2 & 2 & 2 & 2 & 2 & 2 & 2 & 2 & 2 & 2 & 3 & 3\end{array}$

$\begin{array}{llllllllllllllllllllllllllllllll}0 & 1 & 2 & 3 & 4 & 5 & 6 & 7 & 8 & 9 & 0 & 1 & 2 & 3 & 4 & 5 & 6 & 7 & 8 & 9 & 0 & 1 & 2 & 3 & 4 & 5 & 6 & 7 & 8 & 9 & 0 & 1\end{array}$

+-+-+-+-+-+-+-+-+-+-+-+-+-+-+-+-+-+-+-+-+-+-+-+-+-+-+-+-+-+-+-+-+

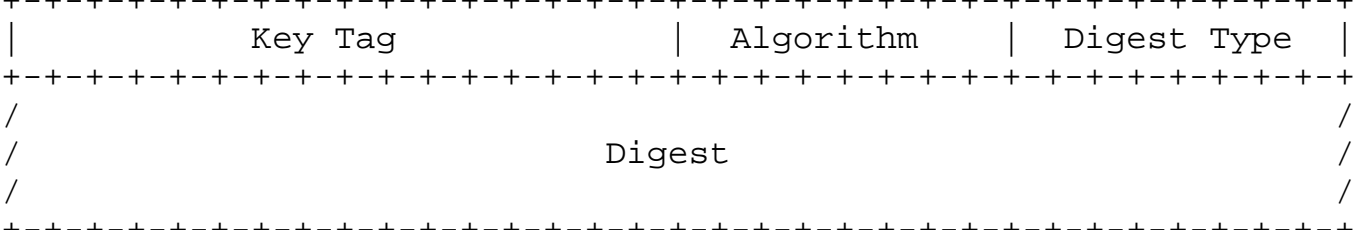

5.1.1. The Key Tag Field

The Key Tag field lists the key tag of the DNSKEY RR referred to by the DS record, in network byte order.

The Key Tag used by the DS RR is identical to the Key Tag used by RRSIG RRs. Appendix B describes how to compute a Key Tag.

5.1.2. The Algorithm Field

The Algorithm field lists the algorithm number of the DNSKEY RR referred to by the DS record.

The algorithm number used by the DS RR is identical to the algorithm number used by RRSIG and DNSKEY RRS. Appendix A.1 lists the algorithm number types. 


\subsubsection{The Digest Type Field}

The DS RR refers to a DNSKEY RR by including a digest of that DNSKEY RR. The Digest Type field identifies the algorithm used to construct the digest. Appendix A.2 lists the possible digest algorithm types.

5.1.4. The Digest Field

The DS record refers to a DNSKEY RR by including a digest of that DNSKEY RR.

The digest is calculated by concatenating the canonical form of the fully qualified owner name of the DNSKEY RR with the DNSKEY RDATA, and then applying the digest algorithm.

digest = digest_algorithm( DNSKEY owner name | DNSKEY RDATA $)$;

"|" denotes concatenation

DNSKEY RDATA = Flags $\mid$ Protocol | Algorithm | Public Key .

The size of the digest may vary depending on the digest algorithm and DNSKEY RR size. As of the time of this writing, the only defined digest algorithm is SHA-1, which produces a 20 octet digest.

\subsection{Processing of DS RRs When Validating Responses}

The DS RR links the authentication chain across zone boundaries, so the DS RR requires extra care in processing. The DNSKEY RR referred to in the DS RR MUST be a DNSSEC zone key. The DNSKEY RR Flags MUST have Flags bit 7 set. If the DNSKEY flags do not indicate a DNSSEC zone key, the DS RR (and the DNSKEY RR it references) MUST NOT be used in the validation process.

5.3. The DS RR Presentation Format

The presentation format of the RDATA portion is as follows:

The Key Tag field MUST be represented as an unsigned decimal integer.

The Algorithm field MUST be represented either as an unsigned decimal integer or as an algorithm mnemonic specified in Appendix A.1.

The Digest Type field MUST be represented as an unsigned decimal integer. 
The Digest MUST be represented as a sequence of case-insensitive hexadecimal digits. Whitespace is allowed within the hexadecimal text.

5.4. DS RR Example

The following example shows a DNSKEY RR and its corresponding DS RR. dskey.example.com. 86400 IN DNSKEY 25635 ( AQOeiiROGOMYkDshWoSKz9Xz fwJr1AYtsmx3TGkJaNXVbfi/ 2pHm822aJ5iI9BMzNXxeYCmZ DRD 9 9WYWYqUSd jMmmAphXdvx egXd/M5+X7OrzKBaMbCVdFLU Uh 6DhweJBjEVv5 f2wwjM9XzC nOf+EPbtG9DMBmAD jEDc2w/r $1 j \mathrm{WVFw}==$

) i key id $=60485$

dskey.example.com. 86400 IN DS 6048551 ( 2BB183AF5F22588179A53B0A 98631FAD1A292118)

The first four text fields specify the name, TTL, Class, and RR type (DS). Value 60485 is the key tag for the corresponding "dskey.example.com." DNSKEY RR, and value 5 denotes the algorithm used by this "dskey.example.com." DNSKEY RR. The value 1 is the algorithm used to construct the digest, and the rest of the RDATA text is the digest in hexadecimal.

6. Canonical Form and Order of Resource Records

This section defines a canonical form for resource records, a canonical ordering of DNS names, and a canonical ordering of resource records within an RRset. A canonical name order is required to construct the NSEC name chain. A canonical RR form and ordering within an RRset are required in order to construct and verify RRSIG RRs.

\subsection{Canonical DNS Name Order}

For the purposes of DNS security, owner names are ordered by treating individual labels as unsigned left-justified octet strings. The absence of a octet sorts before a zero value octet, and uppercase US-ASCII letters are treated as if they were lowercase US-ASCII letters. 
To compute the canonical ordering of a set of DNS names, start by sorting the names according to their most significant (rightmost) labels. For names in which the most significant label is identical, continue sorting according to their next most significant label, and so forth.

For example, the following names are sorted in canonical DNS name order. The most significant label is "example". At this level, "example" sorts first, followed by names ending in "a.example", then by names ending "z.example". The names within each level are sorted in the same way.

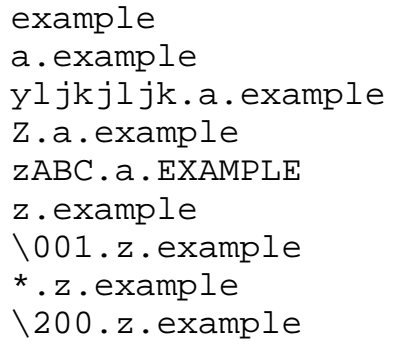

6.2. Canonical RR Form

For the purposes of DNS security, the canonical form of an RR is the wire format of the RR where:

1. every domain name in the RR is fully expanded (no DNS name compression) and fully qualified;

2. all uppercase US-ASCII letters in the owner name of the RR are replaced by the corresponding lowercase US-ASCII letters;

3. if the type of the RR is NS, MD, MF, CNAME, SOA, MB, MG, MR, PTR, HINFO, MINFO, MX, HINFO, RP, AFSDB, RT, SIG, PX, NXT, NAPTR, KX, SRV, DNAME, A6, RRSIG, or NSEC, all uppercase US-ASCII letters in the DNS names contained within the RDATA are replaced by the corresponding lowercase US-ASCII letters;

4. If the owner name of the $R R$ is a wildcard name, the owner name is in its original unexpanded form, including the "*" label (no wildcard substitution); and

5. the RR's TTL is set to its original value as it appears in the originating authoritative zone or the Original TTL field of the covering RRSIG RR. 
6.3. Canonical RR Ordering within an RRset

For the purposes of DNS security, RRs with the same owner name, class, and type are sorted by treating the RDATA portion of the canonical form of each $R R$ as a left-justified unsigned octet sequence in which the absence of an octet sorts before a zero octet.

[RFC2181] specifies that an RRset is not allowed to contain duplicate records (multiple RRs with the same owner name, class, type, and RDATA). Therefore, if an implementation detects duplicate RRs when putting the RRset in canonical form, it MUST treat this as a protocol error. If the implementation chooses to handle this protocol error in the spirit of the robustness principle (being liberal in what it accepts), it MUST remove all but one of the duplicate RR(s) for the purposes of calculating the canonical form of the RRset.

\section{IANA Considerations}

This document introduces no new IANA considerations, as all of the protocol parameters used in this document have already been assigned by previous specifications. However, since the evolution of DNSSEC has been long and somewhat convoluted, this section attempts to describe the current state of the IANA registries and other protocol parameters that are (or once were) related to DNSSEC.

Please refer to [RFC4035] for additional IANA considerations.

DNS Resource Record Types: [RFC2535] assigned types 24, 25, and 30 to the SIG, KEY, and NXT RRs, respectively. [RFC3658] assigned DNS Resource Record Type 43 to DS. [RFC3755] assigned types 46, 47, and 48 to the RRSIG, NSEC, and DNSKEY RRs, respectively. [RFC3755] also marked type 30 (NXT) as obsolete and restricted use of types 24 (SIG) and 25 (KEY) to the "SIG(0)" transaction security protocol described in [RFC2931] and to the transaction KEY Resource Record described in [RFC2930].

DNS Security Algorithm Numbers: [RFC2535] created an IANA registry for DNSSEC Resource Record Algorithm field numbers and assigned values 1-4 and 252-255. [RFC3110] assigned value 5. [RFC3755] altered this registry to include flags for each entry regarding its use with the DNS security extensions. Each algorithm entry could refer to an algorithm that can be used for zone signing, transaction security (see [RFC2931]), or both. Values 6-251 are available for assignment by IETF standards action ([RFC3755]). See Appendix A for a full listing of the DNS Security Algorithm Numbers entries at the time of this writing and their status for use in DNSSEC. 
[RFC3658] created an IANA registry for DNSSEC DS Digest Types and assigned value 0 to reserved and value 1 to SHA-1.

KEY Protocol Values: [RFC2535] created an IANA Registry for KEY Protocol Values, but [RFC3445] reassigned all values other than 3 to reserved and closed this IANA registry. The registry remains closed, and all KEY and DNSKEY records are required to have a Protocol octet value of 3 .

Flag bits in the KEY and DNSKEY RRS: [RFC3755] created an IANA registry for the DNSSEC KEY and DNSKEY RR flag bits. Initially, this registry only contains assignments for bit 7 (the ZONE bit) and bit 15 (the Secure Entry Point flag (SEP) bit; see [RFC3757]). As stated in [RFC3755], bits $0-6$ and 8-14 are available for assignment by IETF Standards Action.

8. Security Considerations

This document describes the format of four DNS resource records used by the DNS security extensions and presents an algorithm for calculating a key tag for a public key. Other than the items described below, the resource records themselves introduce no security considerations. Please see [RFC4033] and [RFC4035] for additional security considerations related to the use of these records.

The DS record points to a DNSKEY RR by using a cryptographic digest, the key algorithm type, and a key tag. The DS record is intended to identify an existing DNSKEY RR, but it is theoretically possible for an attacker to generate a DNSKEY that matches all the DS fields. The probability of constructing a matching DNSKEY depends on the type of digest algorithm in use. The only currently defined digest algorithm is SHA-1, and the working group believes that constructing a public key that would match the algorithm, key tag, and SHA-1 digest given in a DS record would be a sufficiently difficult problem that such an attack is not a serious threat at this time.

The key tag is used to help select DNSKEY resource records efficiently, but it does not uniquely identify a single DNSKEY resource record. It is possible for two distinct DNSKEY RRs to have the same owner name, the same algorithm type, and the same key tag. An implementation that uses only the key tag to select a DNSKEY RR might select the wrong public key in some circumstances. Please see Appendix B for further details. 
The table of algorithms in Appendix A and the key tag calculation algorithms in Appendix B include the RSA/MD5 algorithm for completeness, but the RSA/MD5 algorithm is NOT RECOMMENDED, as explained in [RFC3110].

9. Acknowledgements

This document was created from the input and ideas of the members of the DNS Extensions Working Group and working group mailing list. The editors would like to express their thanks for the comments and suggestions received during the revision of these security extension specifications. Although explicitly listing everyone who has contributed during the decade in which DNSSEC has been under development would be impossible, [RFC4033] includes a list of some of the participants who were kind enough to comment on these documents.

10. References

10.1. Normative References

[RFC1034] Mockapetris, P., "Domain names - concepts and facilities", STD 13, RFC 1034, November 1987.

[RFC1035] Mockapetris, P., "Domain names - implementation and specification", STD 13, RFC 1035, November 1987.

[RFC1982] Elz, R. and R. Bush, "Serial Number Arithmetic", RFC 1982, August 1996 .

[RFC2119] Bradner, S., "Key words for use in RFCs to Indicate Requirement Levels", BCP 14, RFC 2119, March 1997.

[RFC2181] Elz, R. and R. Bush, "Clarifications to the DNS Specification", RFC 2181, July 1997.

[RFC2308] Andrews, M., "Negative Caching of DNS Queries (DNS NCACHE)", RFC 2308, March 1998.

[RFC2536] Eastlake 3rd, D., "DSA KEYs and SIGs in the Domain Name System (DNS)", RFC 2536, March 1999.

[RFC2931] Eastlake 3rd, D., "DNS Request and Transaction Signatures ( SIG(0)s )", RFC 2931, September 2000.

[RFC3110] Eastlake 3rd, D., "RSA/SHA-1 SIGs and RSA KEYs in the Domain Name System (DNS)", RFC 3110, May 2001. 
[RFC3445] Massey, D. and S. Rose, "Limiting the Scope of the KEY Resource Record (RR)", RFC 3445, December 2002.

[RFC3548] Josefsson, S., "The Base16, Base32, and Base64 Data Encodings", RFC 3548, July 2003.

[RFC3597] Gustafsson, A., "Handling of Unknown DNS Resource Record (RR) Types", RFC 3597, September 2003.

[RFC3658] Gudmundsson, O., "Delegation Signer (DS) Resource Record $(\mathrm{RR})$ ", $\mathrm{RFC}$ 3658, December 2003.

[RFC3755] Weiler, S., "Legacy Resolver Compatibility for Delegation Signer (DS)", RFC 3755, May 2004.

[RFC3757] Kolkman, O., Schlyter, J., and E. Lewis, "Domain Name System KEY (DNSKEY) Resource Record (RR) Secure Entry Point (SEP) Flag", RFC 3757, April 2004.

[RFC4033] Arends, R., Austein, R., Larson, M., Massey, D., and S. Rose, "DNS Security Introduction and Requirements", RFC 4033, March 2005.

[RFC4035] Arends, R., Austein, R., Larson, M., Massey, D., and S. Rose, "Protocol Modifications for the DNS Security Extensions", RFC 4035, March 2005.

10.2. Informative References

[RFC2535] Eastlake 3rd, D., "Domain Name system Security Extensions", RFC 2535, March 1999.

[RFC2537] Eastlake 3rd, D., "RSA/MD5 KEYs and SIGs in the Domain Name System (DNS)", RFC 2537, March 1999.

[RFC2539] Eastlake 3rd, D., "Storage of Diffie-Hellman Keys in the Domain Name System (DNS)", RFC 2539, March 1999.

[RFC2930] Eastlake 3rd, D., "Secret Key Establishment for DNS (TKEY RR) ", RFC 2930, September 2000 .

[RFC3845] Schlyter, J., "DNS Security (DNSSEC) NextSECure (NSEC) RDATA Format", RFC 3845, August 2004 . 
Appendix A. DNSSEC Algorithm and Digest Types

The DNS security extensions are designed to be independent of the underlying cryptographic algorithms. The DNSKEY, RRSIG, and DS resource records all use a DNSSEC Algorithm Number to identify the cryptographic algorithm in use by the resource record. The DS resource record also specifies a Digest Algorithm Number to identify the digest algorithm used to construct the DS record. The currently defined Algorithm and Digest Types are listed below. Additional Algorithm or Digest Types could be added as advances in cryptography warrant them.

A DNSSEC aware resolver or name server MUST implement all MANDATORY algorithms.

\section{A.1. DNSSEC Algorithm Types}

The DNSKEY, RRSIG, and DS RRs use an 8-bit number to identify the security algorithm being used. These values are stored in the "Algorithm number" field in the resource record RDATA.

Some algorithms are usable only for zone signing (DNSSEC), some only for transaction security mechanisms (SIG(0) and TSIG), and some for both. Those usable for zone signing may appear in DNSKEY, RRSIG, and DS RRs. Those usable for transaction security would be present in SIG(0) and KEY RRs, as described in [RFC2931].

\begin{tabular}{|c|c|c|c|c|}
\hline Value & Algorithm [Mnemonic] & $\begin{array}{l}\text { Zone } \\
\text { Signing }\end{array}$ & References & Status \\
\hline----- & --------------------- & --------- & ---------- & --------- \\
\hline 0 & reserved & & & \\
\hline 1 & $\mathrm{RSA} / \mathrm{MD} 5$ [RSAMD 5] & $\mathrm{n}$ & [RFC2537] & NOT RECOMMENDED \\
\hline 2 & Diffie-Hellman [DH] & $\mathrm{n}$ & [RFC2539] & - \\
\hline 3 & $\mathrm{DSA} / \mathrm{SHA}-1 \quad[\mathrm{DSA}]$ & $\mathrm{y}$ & [RFC2536] & OPTIONAL \\
\hline 4 & Elliptic Curve [ECC] & & TBA & - \\
\hline 5 & $\mathrm{RSA} / \mathrm{SHA}-1$ [RSASHA1] & $\mathrm{y}$ & [RFC3110] & MANDATORY \\
\hline 252 & Indirect [INDIRECT] & $\mathrm{n}$ & & - \\
\hline 253 & Private [PRIVATEDNS] & $\mathrm{y}$ & see below & OPTIONAL \\
\hline 254 & Private [PRIVATEOID] & $\mathrm{y}$ & see below & OPTIONAL \\
\hline 255 & reserved & & & \\
\hline
\end{tabular}




\section{A.1.1. Private Algorithm Types}

Algorithm number 253 is reserved for private use and will never be assigned to a specific algorithm. The public key area in the DNSKEY $R R$ and the signature area in the RRSIG RR begin with a wire encoded domain name, which MUST NOT be compressed. The domain name indicates the private algorithm to use, and the remainder of the public key area is determined by that algorithm. Entities should only use domain names they control to designate their private algorithms.

Algorithm number 254 is reserved for private use and will never be assigned to a specific algorithm. The public key area in the DNSKEY $R R$ and the signature area in the RRSIG RR begin with an unsigned length byte followed by a BER encoded Object Identifier (ISO OID) of that length. The OID indicates the private algorithm in use, and the remainder of the area is whatever is required by that algorithm. Entities should only use OIDs they control to designate their private algorithms.

\section{A.2. DNSSEC Digest Types}

A "Digest Type" field in the DS resource record types identifies the cryptographic digest algorithm used by the resource record. The following table lists the currently defined digest algorithm types.

$\begin{array}{cl}\text { VALUE } & \text { Algorithm } \\ 0 & \text { Reserved } \\ 1 & \text { SHA-1 } \\ 2-255 & \text { Unassigned }\end{array}$

STATUS<smiles>[CH]1[CH]C1</smiles>

MANDATORY

$-$

Appendix B. Key Tag Calculation

The Key Tag field in the RRSIG and DS resource record types provides a mechanism for selecting a public key efficiently. In most cases, a combination of owner name, algorithm, and key tag can efficiently identify a DNSKEY record. Both the RRSIG and DS resource records have corresponding DNSKEY records. The Key Tag field in the RRSIG and DS records can be used to help select the corresponding DNSKEY RR efficiently when more than one candidate DNSKEY RR is available.

However, it is essential to note that the key tag is not a unique identifier. It is theoretically possible for two distinct DNSKEY RRS to have the same owner name, the same algorithm, and the same key tag. The key tag is used to limit the possible candidate keys, but it does not uniquely identify a DNSKEY record. Implementations MUST NOT assume that the key tag uniquely identifies a DNSKEY RR. 
The key tag is the same for all DNSKEY algorithm types except algorithm 1 (please see Appendix B.1 for the definition of the key tag for algorithm 1). The key tag algorithm is the sum of the wire format of the DNSKEY RDATA broken into 2 octet groups. First, the RDATA (in wire format) is treated as a series of 2 octet groups. These groups are then added together, ignoring any carry bits.

A reference implementation of the key tag algorithm is as an ANSI C function is given below, with the RDATA portion of the DNSKEY RR is used as input. It is not necessary to use the following reference code verbatim, but the numerical value of the Key Tag MUST be identical to what the reference implementation would generate for the same input.

Please note that the algorithm for calculating the Key Tag is almost but not completely identical to the familiar ones-complement checksum used in many other Internet protocols. Key Tags MUST be calculated using the algorithm described here rather than the ones complement checksum.

The following ANSI C reference implementation calculates the value of a Key Tag. This reference implementation applies to all algorithm types except algorithm 1 (see Appendix B.1). The input is the wire format of the RDATA portion of the DNSKEY RR. The code is written for clarity, not efficiency.

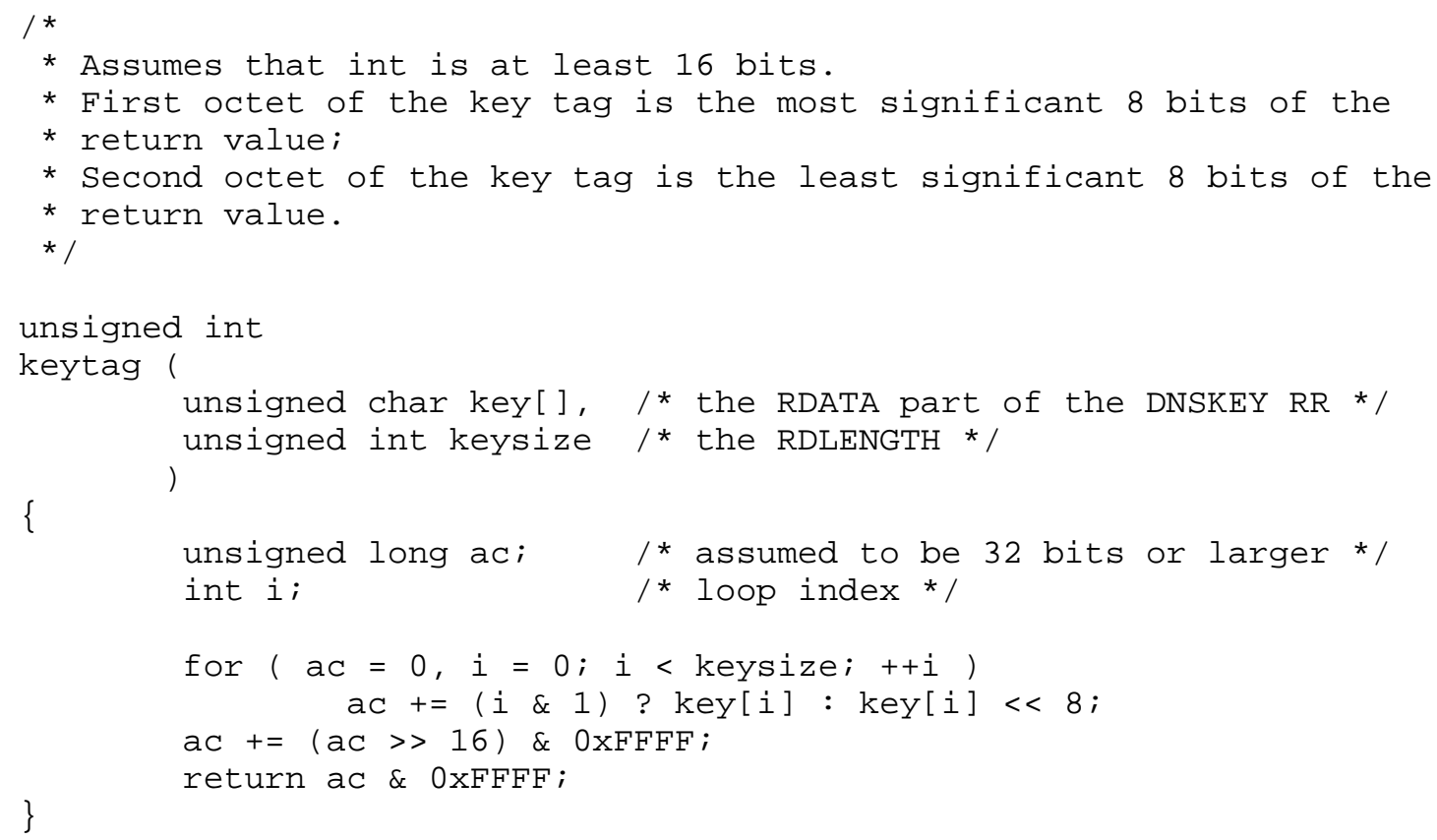


B.1. Key Tag for Algorithm 1 (RSA/MD5)

The key tag for algorithm 1 (RSA/MD5) is defined differently from the key tag for all other algorithms, for historical reasons. For a DNSKEY RR with algorithm 1, the key tag is defined to be the most significant 16 bits of the least significant 24 bits in the public key modulus (in other words, the 4th to last and 3rd to last octets of the public key modulus).

Please note that Algorithm 1 is NOT RECOMMENDED. 


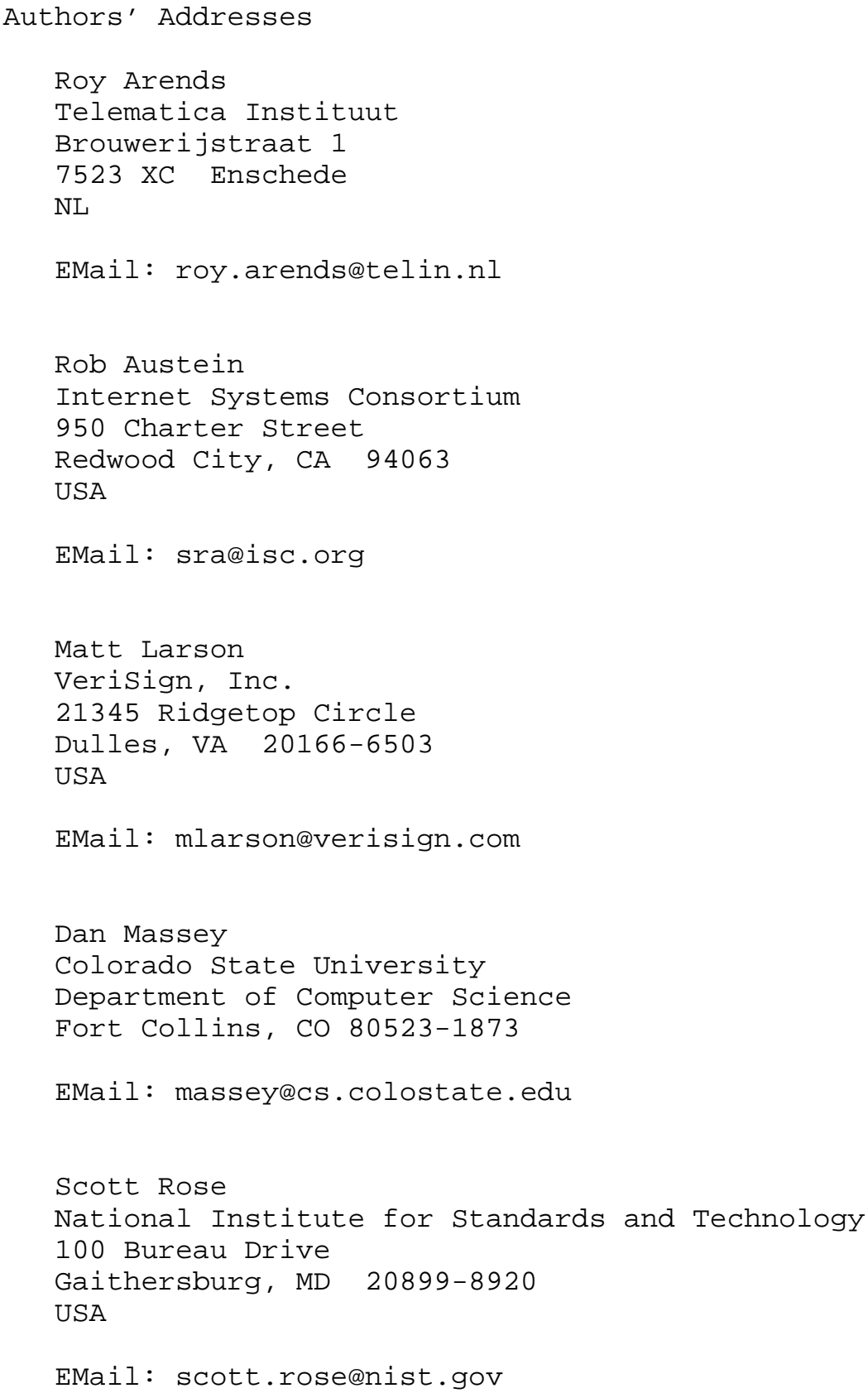


Full Copyright statement

Copyright (C) The Internet Society (2005).

This document is subject to the rights, licenses and restrictions contained in BCP 78, and except as set forth therein, the authors retain all their rights.

This document and the information contained herein are provided on an "AS IS" basis and THE CONTRIBUTOR, THE ORGANIZATION HE/SHE REPRESENTS OR IS SPONSORED BY (IF ANY), THE INTERNET SOCIETY AND THE INTERNET ENGINEERING TASK FORCE DISCLAIM ALL WARRANTIES, EXPRESS OR IMPLIED, INCLUDING BUT NOT LIMITED TO ANY WARRANTY THAT THE USE OF THE INFORMATION HEREIN WILL NOT INFRINGE ANY RIGHTS OR ANY IMPLIED WARRANTIES OF MERCHANTABILITY OR FITNESS FOR A PARTICULAR PURPOSE.

Intellectual Property

The IETF takes no position regarding the validity or scope of any Intellectual Property Rights or other rights that might be claimed to pertain to the implementation or use of the technology described in this document or the extent to which any license under such rights might or might not be available; nor does it represent that it has made any independent effort to identify any such rights. Information on the procedures with respect to rights in RFC documents can be found in BCP 78 and BCP 79 .

Copies of IPR disclosures made to the IETF Secretariat and any assurances of licenses to be made available, or the result of an attempt made to obtain a general license or permission for the use of such proprietary rights by implementers or users of this specification can be obtained from the IETF on-line IPR repository at http://www.ietf.org/ipr.

The IETF invites any interested party to bring to its attention any copyrights, patents or patent applications, or other proprietary rights that may cover technology that may be required to implement this standard. Please address the information to the IETF at ietfipreietf.org.

Acknowledgement

Funding for the RFC Editor function is currently provided by the Internet Society. 